Supporting information for

\title{
Tailored Graphene Oxide Membranes for the Separation of Ions and Molecules
}

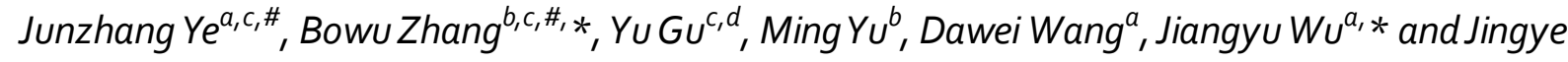
$L i^{b, c}, *$

${ }^{a}$ College of Materials Science and Engineering, Wuhan Institute of Technology, No.206 Guanggu 1st Road, Donghu New \& High Technology Development Zone, Wuhan, Hubei Province, 430205, China

${ }^{b}$ The Education Ministry Key Lab of Resource Chemistry, Shanghai Key Lab of Rare Earth Functional Materials, College of Chemistry and Materials Science, Shanghai Normal University, No. 100 Guilin Road, Shanghai, 200234, China

'Shanghai Institute of Applied Physics, Chinese Academy of Sciences, No. 2019 Jialuo Road, Shanghai, 201800, China

d University of Chinese Academy of Sciences, No. 19A, Yuquan Road, Beijing, 100049, China. *Correspondence authors.

E-mail addresses: zhangbowu@shnu.edu.cn (Dr. B.Zhang); jyli@shnu.edu.cn (Prof. J. Li); wujy@wit.edu.cn (Prof. J.Wu)

${ }^{\#}$ These authors contributed to this work equally. 


\section{Experimental}

\section{S1. Reagents and Materials}

Flaky graphite was purchased from Sigma-Aldrich Co. LLC. The sulfuric acid $\left(\mathrm{H}_{2} \mathrm{SO}_{4}\right)$, nitric acid $\left(\mathrm{HNO}_{3}\right)$, hydrochloric acid $(\mathrm{HCl})$, phosphorus pentoxide $\left(\mathrm{P}_{2} \mathrm{O}_{5}\right)$, potassium persulfate $\left(\mathrm{K}_{2} \mathrm{~S}_{2} \mathrm{O}_{8}\right)$, potassium permanganate $\left(\mathrm{KMnO}_{4}\right)$, hydrogen peroxide solution $\left(\mathrm{H}_{2} \mathrm{O}_{2}\right)$, sodium hydroxide $(\mathrm{NaOH})$, sodium chloride $(\mathrm{NaCl})$, Calcium chloride $\left(\mathrm{CaCl}_{2}\right)$, potassium chloride $(\mathrm{KCl})$, magnesium chloride hexahydrate $\left(\mathrm{MgCl}_{2} \cdot 6 \mathrm{H}_{2} \mathrm{O}\right)$, were all purchased from Sinopharm Chemical Reagent Co., Ltd. All reagents were used without any purification. 1-allyl-3-vinylimidazolium chloride ([AVIM]Cl) was purchased from Lanzhou Institute of Chemical Physics (LICP) of the Chinese Academy of Sciences (CAS), whose molecular structures are shown as Figure $\mathrm{S}_{3}$. The de-ionized water (DI) used for all the experiment in this paper was taken from Milli-Q purification system (Millipore, USA). Cellulose acetate (CA) membranes (pore size, $0.45 \mu \mathrm{m}$ ) were purchased from Shanghai Xingya Purifying Material Factory.

\section{S2. Preparation of graphene oxide sheets}

The graphene oxide sheets were prepared by the modified Hummers' method. In detail, a certain amount of flaky graphite was poured into the flask, and $\mathrm{HNO}_{3}$ was added for pretreatment for 24 hours. The graphite powder was then placed in a muffle furnace for high temperature treatment to prepare expanded graphite. The expanded graphite was preoxidized with $\mathrm{HNO}_{3}, \mathrm{H}_{2} \mathrm{SO}_{4}, \mathrm{~K}_{2} \mathrm{~S}_{2} \mathrm{O}_{8}, \mathrm{P}_{2} \mathrm{O}_{5}$ and the product was dispersed in water to collect the precipitate. After drying, the pre-oxidized graphene oxide is further oxidized with $\mathrm{KMnO}_{4}$ and $\mathrm{H}_{2} \mathrm{SO}_{4}$ and $30 \% \mathrm{H}_{2} \mathrm{O}_{2}$. Finally, the graphene oxide solution was dialyzed with deionized water until near neutral, and the concentration of the graphene oxide dispersion prepared was $3.7 \mathrm{mg} / \mathrm{mL}$. 


\section{Table S1}

\begin{tabular}{|c|c|}
\hline Species & Hydrated dimeter $(\mathrm{nm})$ \\
\hline $\mathrm{K}^{+}$ & $0.662^{1-2}$ \\
\hline $\mathrm{Na}^{+}$ & $0.716^{1-2}$ \\
\hline $\mathrm{Ca}^{2+}$ & $0.824^{1-2}$ \\
\hline $\mathrm{Mg}^{2+}$ & $0.856^{1-2}$ \\
\hline $\mathrm{Glycerol}$ & $0.93^{3}$ \\
\hline $\mathrm{Fe}^{3^{+}}$ & $0.96^{1}$ \\
\hline $\mathrm{MO}$ & $0.99^{4}$ \\
\hline $\mathrm{RhB}$ & $1.232^{4}$ \\
\hline
\end{tabular}

\section{References:}

(1) Tansel, B., Significance of thermodynamic and physical characteristics on permeation of ions during membrane separation: Hydrated radius, hydration free energy and viscous effects. Sep. Purif. Technol. 2012, 86, 119-126.

(2) Qian, Y.; Zhou, C.; Huang, A., Cross-linking modification with diamine monomers to enhance desalination performance of graphene oxide membranes. Carbon 2018, 136, 28-37.

(3) Joshi, R. K.; Carbone, P.; Wang, F. C.; Kravets, V. G.; Su, Y.; Grigorieva, I. V.; Wu, H. A.; Geim, A. K.; Nair, R. R., Precise and Ultrafast Molecular Sieving Through Graphene Oxide Membranes. Science 2014, 343, 752-754.

(4) Akbari, A.; Sheath, P.; Martin, S. T.; Shinde, D. B.; Shaibani, M.; Banerjee, P. C.; Tkacz, R.; Bhattacharyya, D.; Majumder, M., Large-area graphene-based nanofiltration membranes by shear alignment of discotic nematic liquid crystals of graphene oxide. Nat. Commun. 2016, 7, 10891. 


\section{Figures}

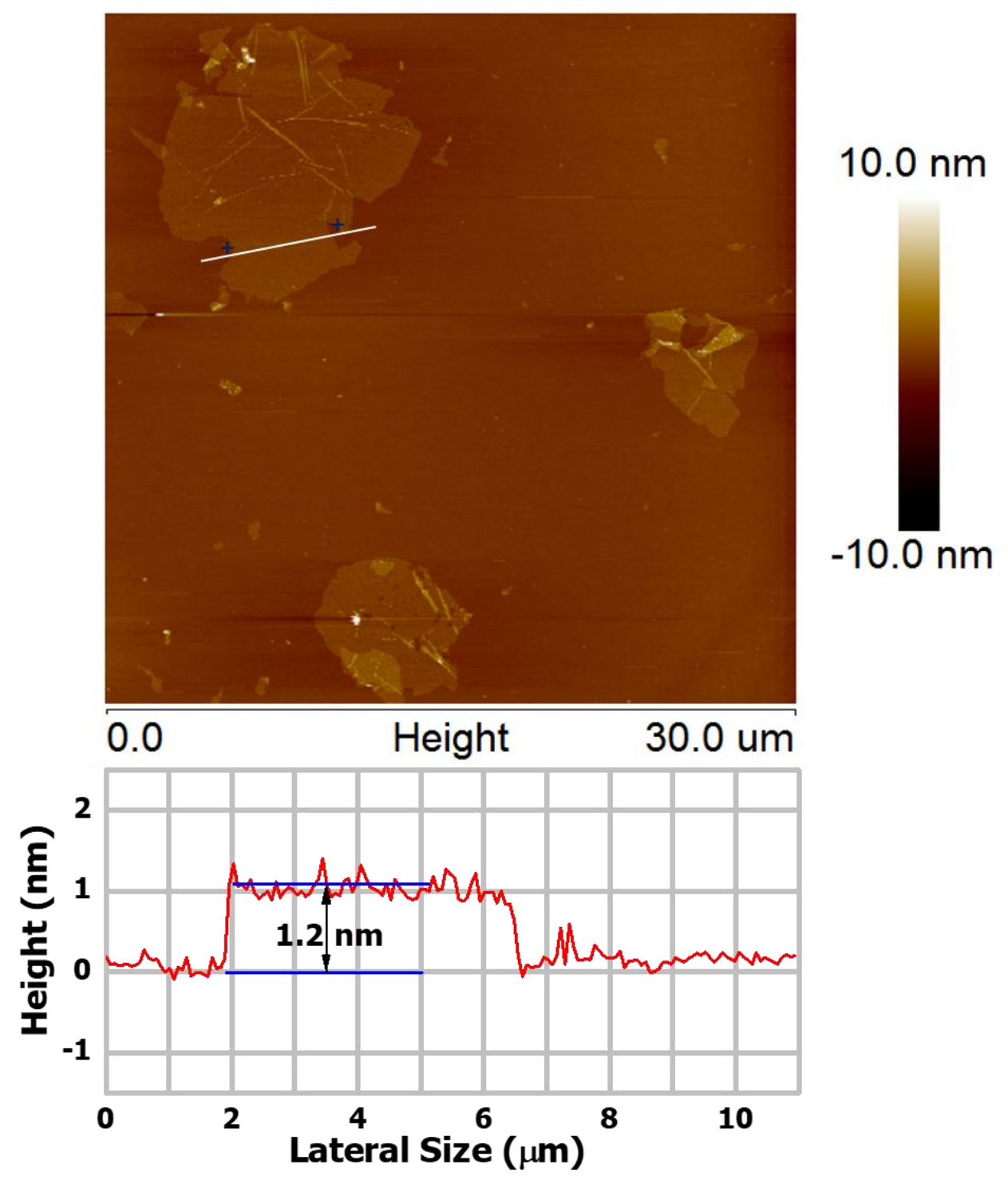

Figure S1. The AFM image of the GO sheets as prepared. 


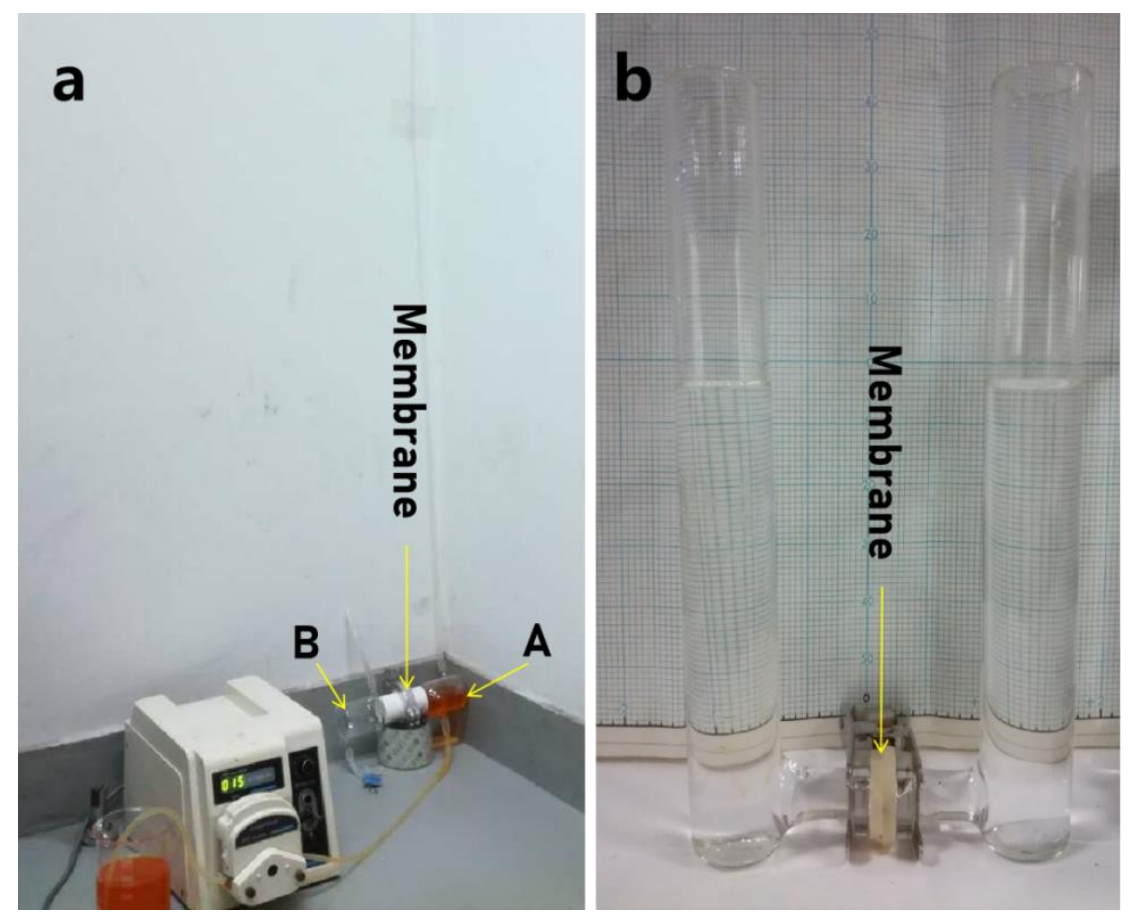

Figure S2. The setups of hydrostatic head test (a) and permeation test (b).

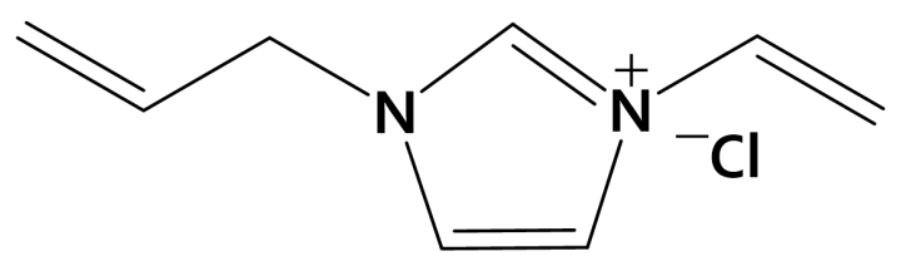

1-allyl-3-vinylimidazolium chloride ([AVIM]Cl)

Figure S3. The chemical structure of $[\mathrm{AVIM}] \mathrm{Cl}$. 

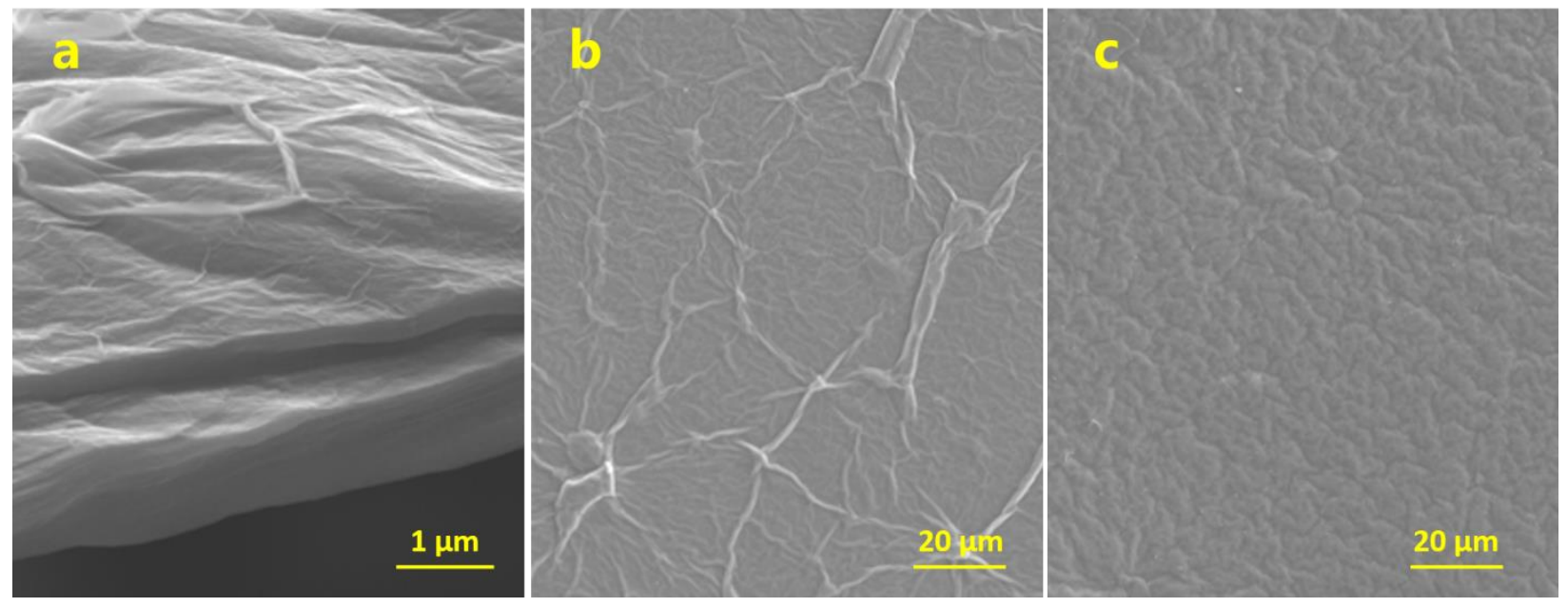

Figure S4. SEM images of (a) the cross-section of GO membrane, the surface of (b) GO membrane and (c) i-AVG membrane.

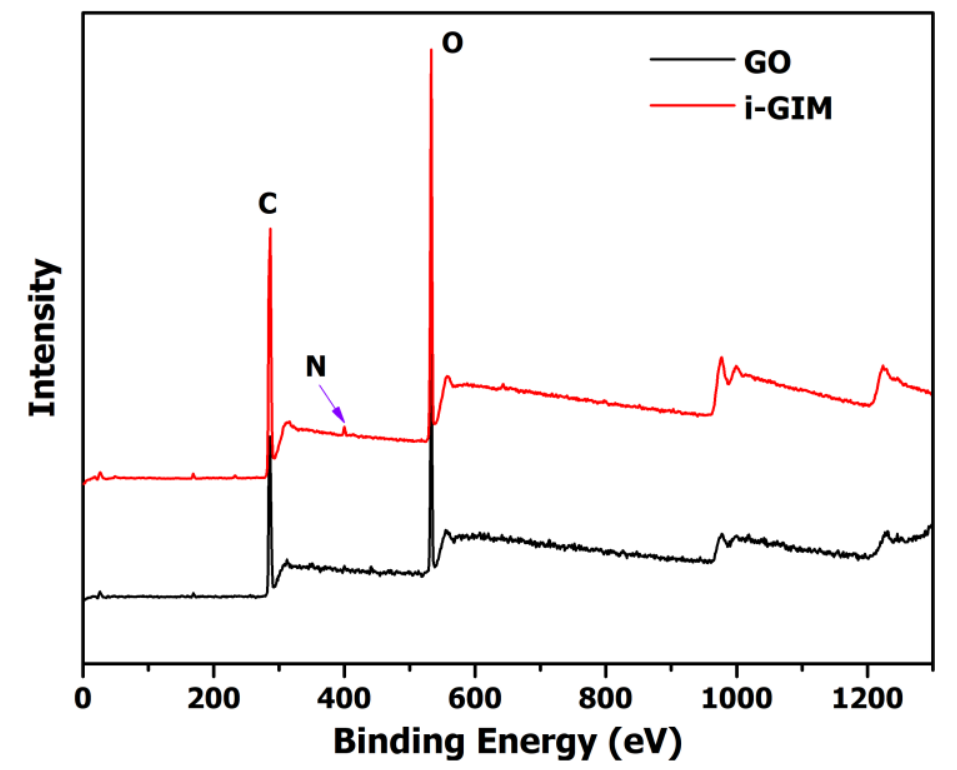

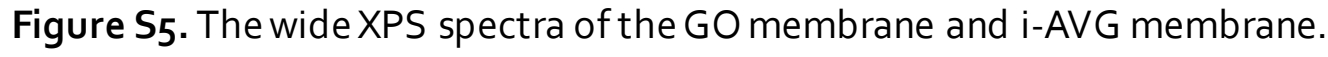



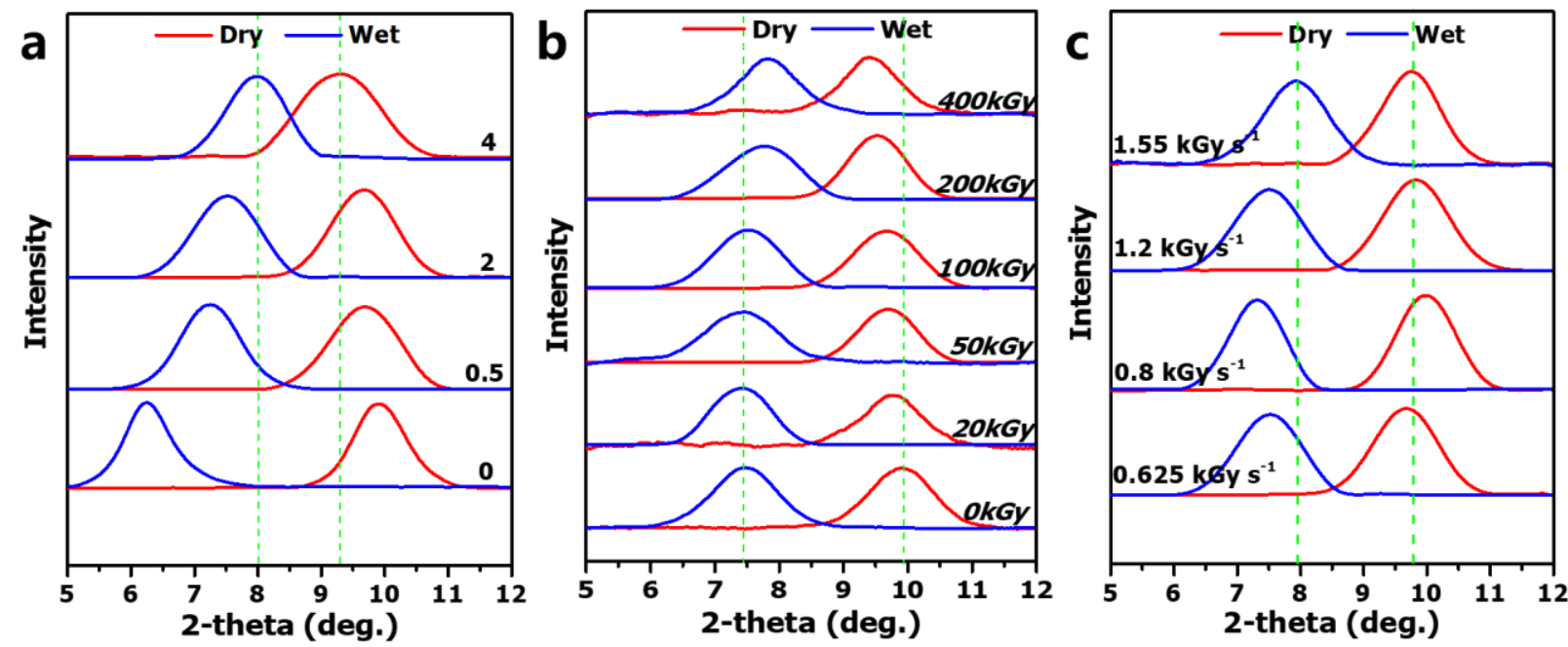

Figure S6. The XRD patterns of (a) i-AVG membranes (100 kGy, $0.625 \mathrm{KGy} \mathrm{s}^{-1}$ ) prepared from mixing dispersions with different mass ratio of $[A V I M] C l / G O$, (b) i-AVG membranes $\left([\mathrm{AVIM}] \mathrm{Cl} / \mathrm{GO}=2,0.625 \mathrm{kGy} \mathrm{s}^{-1}\right.$ ) over different absorbed dose at $0.625 \mathrm{kGy} \mathrm{s}^{-1}$ of dose rate and (c) i-AVG membranes ([AVIM]Cl/GO=2, $100 \mathrm{kGy}$ ) over different dose rate.
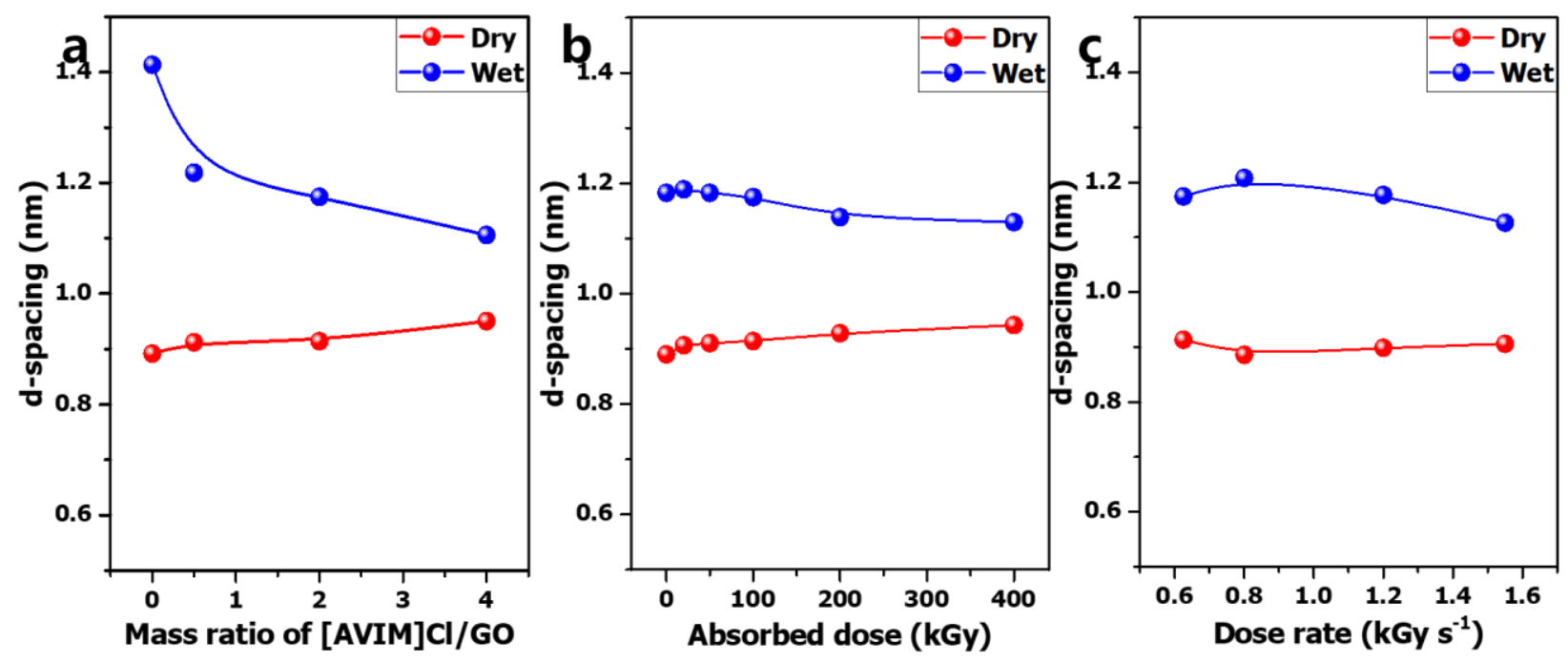

Figure S7. The d-spacing of dry and wet (a) i-AVG membranes (100 kGy, $0.625 \mathrm{kGy} \mathrm{s}^{-1}$ ) prepared from mixing dispersions with different mass ratio of $[A V I M] C l / G O$, (b) i-AVG membranes $\left([\mathrm{AVIM}] \mathrm{Cl} / \mathrm{GO}=2,0.625 \mathrm{kGy} \mathrm{s}^{-1}\right.$ ) over different absorbed dose at $0.625 \mathrm{kGy} \mathrm{s}^{-1}$ of dose rate and (c) i-AVG membranes ([AVIM]Cl/GO=2, $100 \mathrm{kGy}$ ) over different dose rate. 